\title{
Sea-state modification and heaving float interaction factors from physical modelling of arrays of wave energy converters
}

\author{
V. Stratigaki, ${ }^{1, a)}$ P. Troch, ${ }^{1}$ T. Stallard, ${ }^{2}$ D. Forehand, ${ }^{3}$ M. Folley, ${ }^{4}$ \\ J. P. Kofoed, ${ }^{5}$ M. Benoit, ${ }^{6}$ A. Babarit, ${ }^{7}$ M. Vantorre, ${ }^{1}$ and J. Kirkegaard ${ }^{8}$ \\ ${ }^{1}$ Department of Civil Engineering, Ghent University, Ghent 9052, Belgium \\ ${ }^{2}$ School of Mechanical Aerospace and Civil Engineering, University of Manchester, \\ Manchester M13 9PL, United Kingdom \\ ${ }^{3}$ School of Engineering, Institute for Energy Systems, The University of Edinburgh, \\ Edinburgh EH9 3JL, United Kingdom \\ ${ }^{4}$ School of Planning, Architecture \& Civil Engineering, Queen's University Belfast, \\ Belfast BT9 5AG, United Kingdom \\ ${ }^{5}$ Department of Civil Engineering, Wave Energy Research Group, Aalborg University, \\ Aalborg 9000, Denmark \\ ${ }^{6}$ IRPHE \& Ecole Centrale Marseille, Institut de Recherche sur les Phenomenes Hors \\ Equilibre, UMR 7342 (CNRS, Aix-Marseille Universite, Ecole Centrale Marseille), \\ 13384 Marseille Cedex 13, France \\ ${ }^{7}$ Ocean Energy and Ocean Waves Group, LHEAA Lab-CNRS, Ecole Centrale de Nantes, \\ Nantes 44321, France \\ ${ }^{8}$ Ocean Ports and Offshore Technology, DHI, Hørsholm 2970, Denmark
}

Wave energy converters (WECs) extract energy from ocean waves and have the potential to produce a significant amount of electricity from a renewable resource. However, large "WEC farms" or "WEC arrays" (composed of a large number of individual WECs) are expected to exhibit "WEC array effects". These effects represent the impact of the WECs on the wave climate at an installation site, as well as on the overall power absorption of the WEC array. Tests have been performed in the Shallow Water Wave Basin of DHI (Denmark) to study such "WEC array effects". Large arrays of up to 25 heaving point absorber type WECs have been tested for a range of geometric layout configurations and wave conditions. Each WEC consists of a buoy with a diameter of $0.315 \mathrm{~m}$. Power take-off was modeled by realizing friction based energy dissipation through damping of the WECs' motion. The produced database is presented: WEC response, wave induced forces on the WECs, and wave field modifications have been measured. A first understanding of WEC array effects is obtained. This unique experimental set-up of up to 25 individual WEC units in an array layout, placed in a large wave tank, is at present the largest setup of its kind studying the important WEC array effects. The data obtained from these experimental tests will be very useful for vali-dation and extension of numerical models. This model validation will enable opti-mization of the geometrical layout of WEC arrays for realistic wave farm applications and reduction of the cost of energy from wave energy systems.

\section{INTRODUCTION}

The shrinking reserves of fossil fuels in combination with the increasing energy demand have enhanced the interest in sustainable and renewable energy sources, including wave energy. In order to extract a considerable amount of wave power, large numbers of Wave Energy

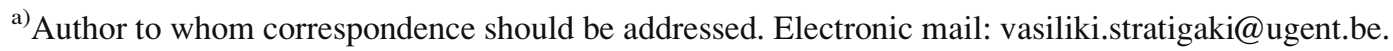


Converters (abbreviated as WECs) will have to be arranged in arrays (or "farms" or "parks") using a particular geometric layout. The operational behaviour of an individual WEC may have a positive or negative effect on the power absorption of the neighbouring WECs in the array (so-called "intra-array interactions"). As a result of the interaction between the WECs within an array, the overall power absorption is affected. In addition, the wave height behind a large array of WECs is modified which may influence neighbouring farms, other users in the sea, or even at the coastline (so-called "extra-array effects"). Both the "intra-array interactions" between WECs in a wave farm and their "extra array effects" on their environment will be referred to as "WEC array effects" in this paper.

The design of a WEC array and the study of WEC array effects require both numerical modelling and experimental testing of a physical scale model. Numerical modelling is very efficient, especially in the first design stages, but model tests in a wave basin or wave flume are necessary since the numerical methods suffer from important limitations. ${ }^{3}$ Numerical studies on both small and large WEC arrays have already been performed, and have provided insight into the magnitude and extent of WEC array effects ${ }^{1,2}$ for idealized conditions and configurations. The hydrodynamic behavior of WECs is modeled by a wide range of numerical methods. As recently reviewed, ${ }^{3,4}$ each method has its drawbacks and assets. For instance, certain models are more suitable for studying intra-array interactions in the near-field, whilst others are preferable for investigating extra-array effects in the far-field of the WEC array. However, there has been very limited validation of these numerical models using physical scale models of large WEC arrays.

Individual $^{5}$ or pairs of $\mathrm{WECs}^{6}$ have been widely experimentally studied, based both on complex and simple geometries, operational principles, and power take-off systems (abbreviated as PTO-systems). Moreover, a small number of wave energy converters have been tested under real sea conditions. ${ }^{7}$ Only a few laboratory studies of small WEC arrays have been conducted using less than $12 \mathrm{WECs}$, including response to regular waves, ${ }^{8}$ power output and response in irregular waves ${ }^{9}$ and wave spectra changes across the array. ${ }^{10}$ Within the UK Supergen Marine and the EU Hydralab III programmes, tests have been conducted of a WEC array of five oscillating water columns interconnected by mooring lines. ${ }^{11}$ As part of the PerAWaT project, several studies of wave energy converter arrays have been conducted, both of idealized geometries $^{12}$ and scale models of WEC systems under development by private companies.

To our knowledge, no experimental studies are publicly available or reported in literature detailing WEC response, power output, and wave field modifications due to large WEC arrays. However, such data are essential for evaluation of the accuracy of the used numerical tools, their validation, as well as for their further development and improvement. This knowledge is necessary to improve understanding of the fundamental processes influencing wave conditions down- and up-wave of wave energy converter arrays, and to optimize of the array lay-outs for realistic wave farm applications.

The need for experiments with large WEC arrays has led to the research project "WECwakes", funded by the EU-FP7-HYDRALAB-IV programme. Within this project, experiments have been performed in the Shallow Water Wave basin of DHI in Denmark (formerly known as Danish Hydraulics Institute) on large arrays of point absorber type WECs with the intention to study WEC array effects for a range of array geometric configurations and wave conditions. The experimental set-up has been designed to simulate the real impact of WEC arrays on the wave climate by using a simple concept of energy extraction from the incoming waves.

As a result of the literature gap regarding WEC array experiments, the following questions had still to be addressed:

- what is the positive or negative effect on power absorption when the number of WECs in an array is increasing?

- what is the magnitude and the impact of the extra-array effects on other users in the sea and the coastline?

- what is the extent of the region of wave field modifications downwave of the WEC arrays?

- what is the influence of the geometric configuration of the WECs within the array, on the extraarray effects? 
- what is the influence of the WEC number on net power absorption by an array?

- what is the influence of the lateral and longitudinal spacing between the WECs on net power absorption by an array?

Consequently, the main objective of the research performed within the "WECwakes" project was to provide insight to the aforementioned questions and, therefore, to improve the understanding of WEC array effects, i.e., the change of wave conditions due to energy extraction by WEC arrays and interactions between the WECs of an array. Knowledge of both the behaviour of an individual WEC affecting its neighbours (intra-array interactions) and the effects downwave of a large array (extra-array effects) is very important in the design of WEC arrays.

In order to achieve the main research objective, the following more detailed objectives have been defined:

- To understand the behaviour of WEC arrays under varying wave conditions;

- To determine the intra-array interactions between the WECs;

- To determine the extra-array effects: to measure and detect the dimensions of wave field changes due to WEC arrays and their impact;

- To quantify the effect on power absorption, by:

(i) changing the lateral, w, and longitudinal, 1, spacing between the WECs within an array,

(ii) changing the number of WECs within an array,

(iii) modifying the WEC array geometric layout.

- To provide an experimental database for validation of numerical models, used by researchers worldwide for wave propagation through WEC arrays and wave-WEC interaction.

This experimental set-up of 25 individual WEC units in an array layout and placed in this large wave tank is at present the largest set-up of its kind worldwide, studying the important impacts on power absorption and wave conditions of WEC array effects. Most importantly, the "WECwakes" database is extensive and non-confidential, accessible to the research community as specified under the HYDRALAB rules, and can be extrapolated to floating structures/platforms, stationary cylinders under wave action, etc., for understanding of, e.g., wave impact on the cylinders and wave field modifications around them.

A detailed overview of the design and conduct of the experiments and of the WECwakes database is given in Sections II-IV. In Sections V and VI, experimental results are presented for the wave field modification caused by 25 heaving WECs in array geometric configurations (for irregular long-crested waves), as well as the paper discussion on the presented findings and on the "WECwakes" database.

\section{EXPERIMENTAL SET-UP}

\section{A. Description of an individual wave energy converter}

The WEC designed and employed for the "WECwakes" project allowed large scale experiments by testing 28 different WEC (array) configurations in the wave basin of DHI. The wave device is generic and is based on simple operating principles, regarding both response and PTO-system (Fig. 5(a)). It has to be noted that the developed WEC unit and especially its PTO-system do not represent a new WEC concept. The PTO-system, for instance, has been designed only to simulate and realize power extraction from the waves and not to represent a new PTO principle. Moreover, a series of experiments with (i) an individual WEC and (ii) small WEC arrays composed of up to 4 WEC units has been performed within the framework of the preparation of the "WECwakes" experiments.

The WEC is based on the point absorber principle, is composed of a buoy (diameter, $\mathrm{D}=31.5 \mathrm{~cm}$ ) designed to heave along a vertical shaft only, and can thus be modeled as a single degree of freedom system. Energy absorption through the WEC's PTO-system, which is based on friction brakes comprising PTFE blocks (made of polytetrafluoroethylene material, commonly known as "Teflon") and 4 linear springs, is modelled by realising energy dissipation through damping of the WEC's heave motion. 
The WEC geometry and PTO-system principle are generic and the WEC can therefore be used for any study of WEC array effects of similar WECs. The developed WEC is easy to operate and feasible to manufacture in large numbers, economically and practically, and is simple to model analytically and numerically.

Details on the WEC unit development, evaluation, and experimental study for the preparation of the WECwakes project are presented by Stratigaki et al. ${ }^{13-15}$ The preparatory testing of the few first prototype WEC units was followed by the construction of 25 identical WEC units, realized at the workshop of Ghent University. Preliminary results for WEC response amplitude operator (RAO) and power output show reasonable agreement between measured response for individual WECs, and power output and WEC response predicted using a linear time domain model. ${ }^{16}$ Moreover, detailed results regarding the efficiency of the WEC (arrays) are presented by Troch et al. ${ }^{16}$ and Stratigaki. ${ }^{15}$

\section{B. The shallow water wave basin (DHI)}

The "WECwakes" tests have been performed in the Shallow Water Wave Basin of DHI (Hørsholm, Denmark). In Fig. 1, an illustration of the wave basin is shown $(25.0 \mathrm{~m}$ long and $35.0 \mathrm{~m}$ wide, with an overall depth of $0.8 \mathrm{~m}$ ). Forty-four piston type wave paddles generate waves at one end of the wave basin (Fig. 2) along a length of $22 \mathrm{~m}$, while a stone gravel beach provides energy absorption at the opposite end of the wave basin.

For the installation of the WECs in the wave basin, a supporting structure has been used comprising the WEC metal gravity bases, the WEC steel vertical shafts, and a connecting steel frame at the top of the WEC shafts, as shown in Fig. 3.

\section{The WECwakes experimental arrangement}

The complexity of the tested WEC array layouts increases gradually. The experiments start with the testing of individual WEC units at different locations within the wave basin. Furthermore, different WEC arrays have been tested, with various geometric configurations and different/increasing WEC numbers. In Fig. 4, a plan view of the configuration comprising the $5 \times 5$-WEC aligned array is presented, showing the general experimental arrangement in the wave basin and the locations of the wave gauges used for this specific configuration.

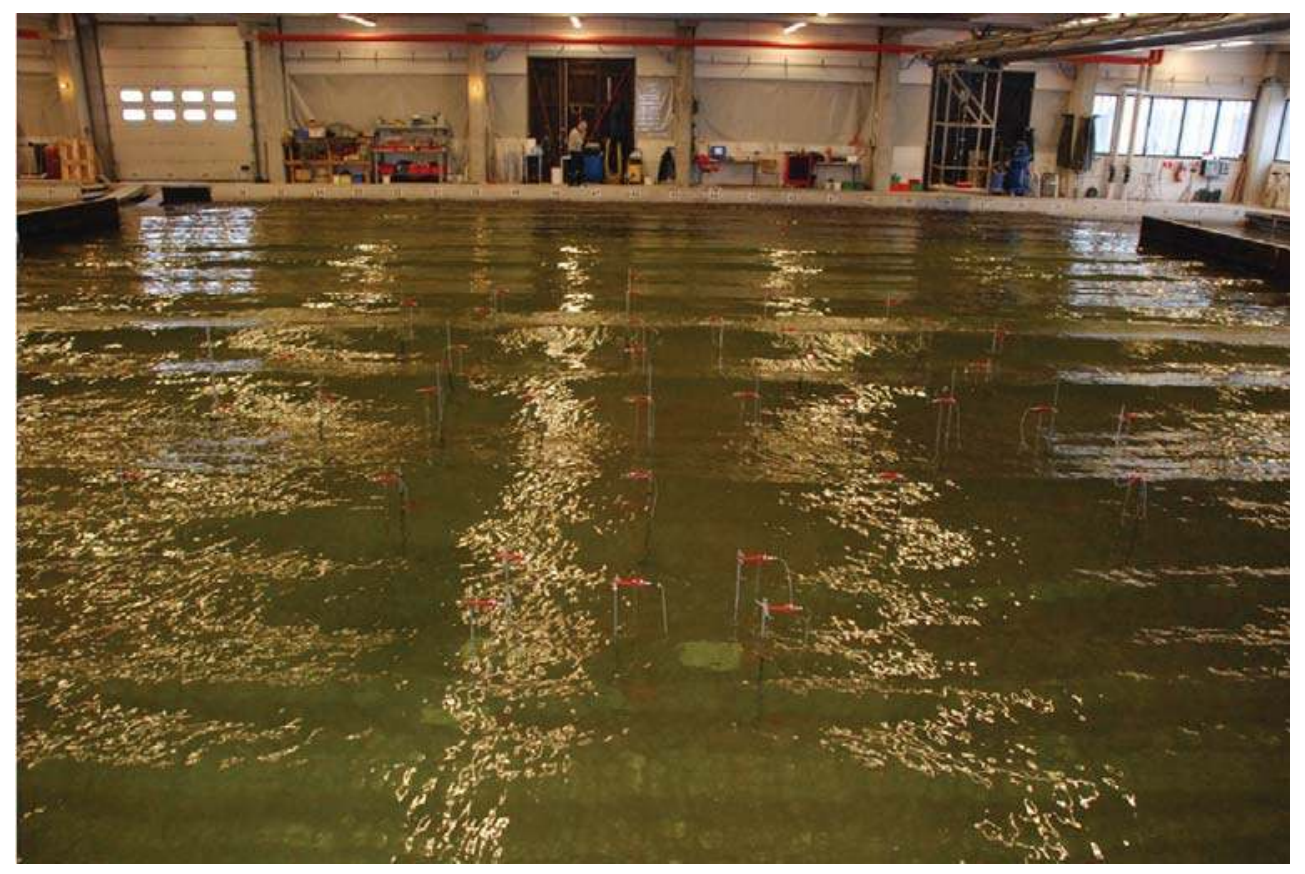

FIG. 1. Shallow Water Wave Basin at DHI (Hørsholm, Denmark). View from a location behind the wave generator, while taking wave height measurements (sea-states characterization) using a network of resistive wave gauges in the empty wave basin. 


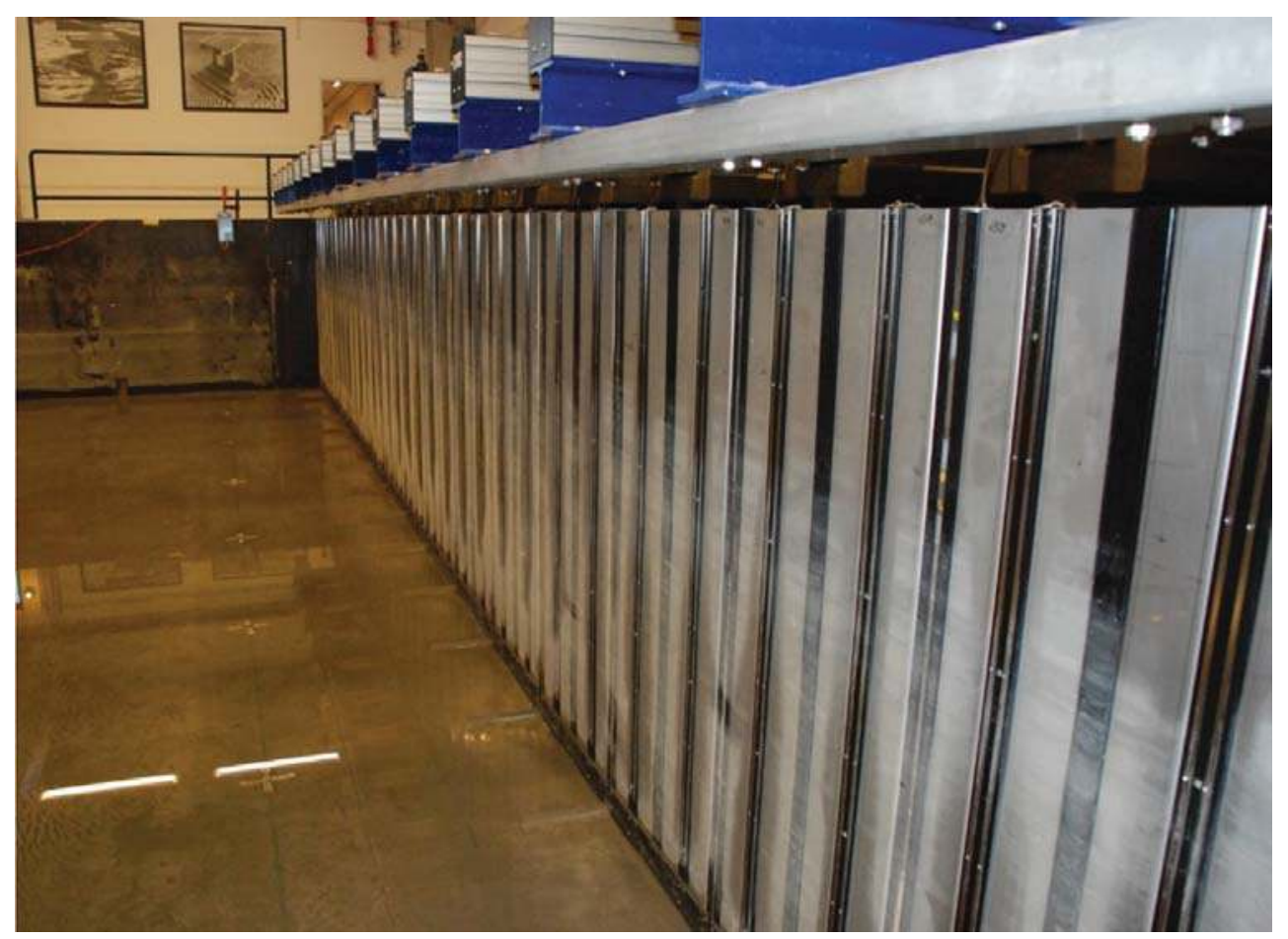

FIG. 2. Wave generator at the Shallow Water Wave Basin of DHI (Hørsholm, Denmark). View to the wave paddles from the side of the wave basin.

As the wave generator does not extend across the entire basin width, vertical guide walls have been installed in order to avoid diffraction of the generated waves to either side of the basin. This technique results to a larger "effective" domain within the wave basin. Moreover, it simplifies the numerical treatment of the experimental set-up, using, e.g., fully reflective boundaries for simulating the guide walls.

\section{INSTRUMENTATION AND ACQUIRED DATA}

\section{A. Wave field measurements}

A network of 41 resistive Wave Gauges (abbreviated as WGs) has been used to take surface elevation measurements $(\eta(\mathrm{t}))$ at specific locations throughout the wave basin. Moreover, a

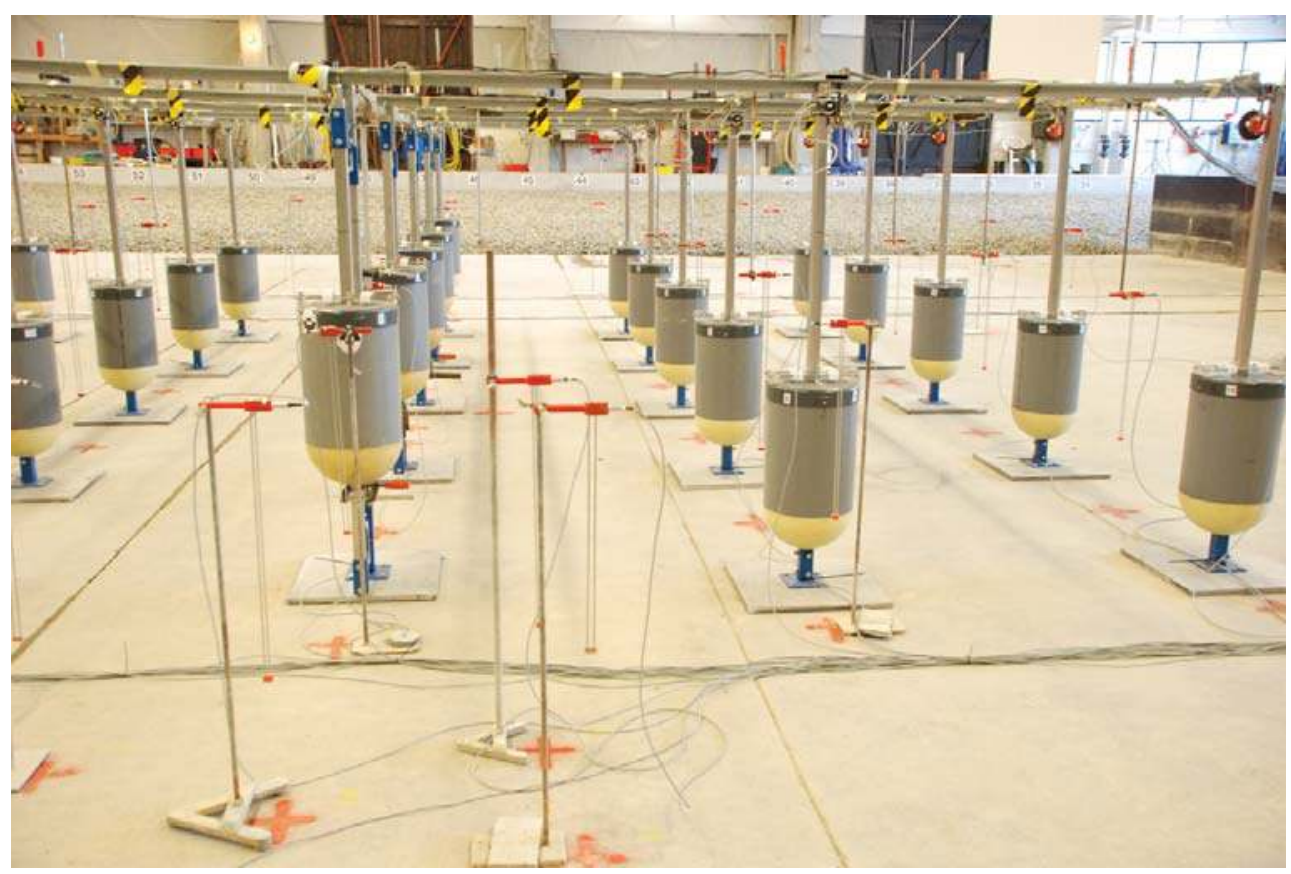

FIG. 3. The support structures for the WEC arrays. 


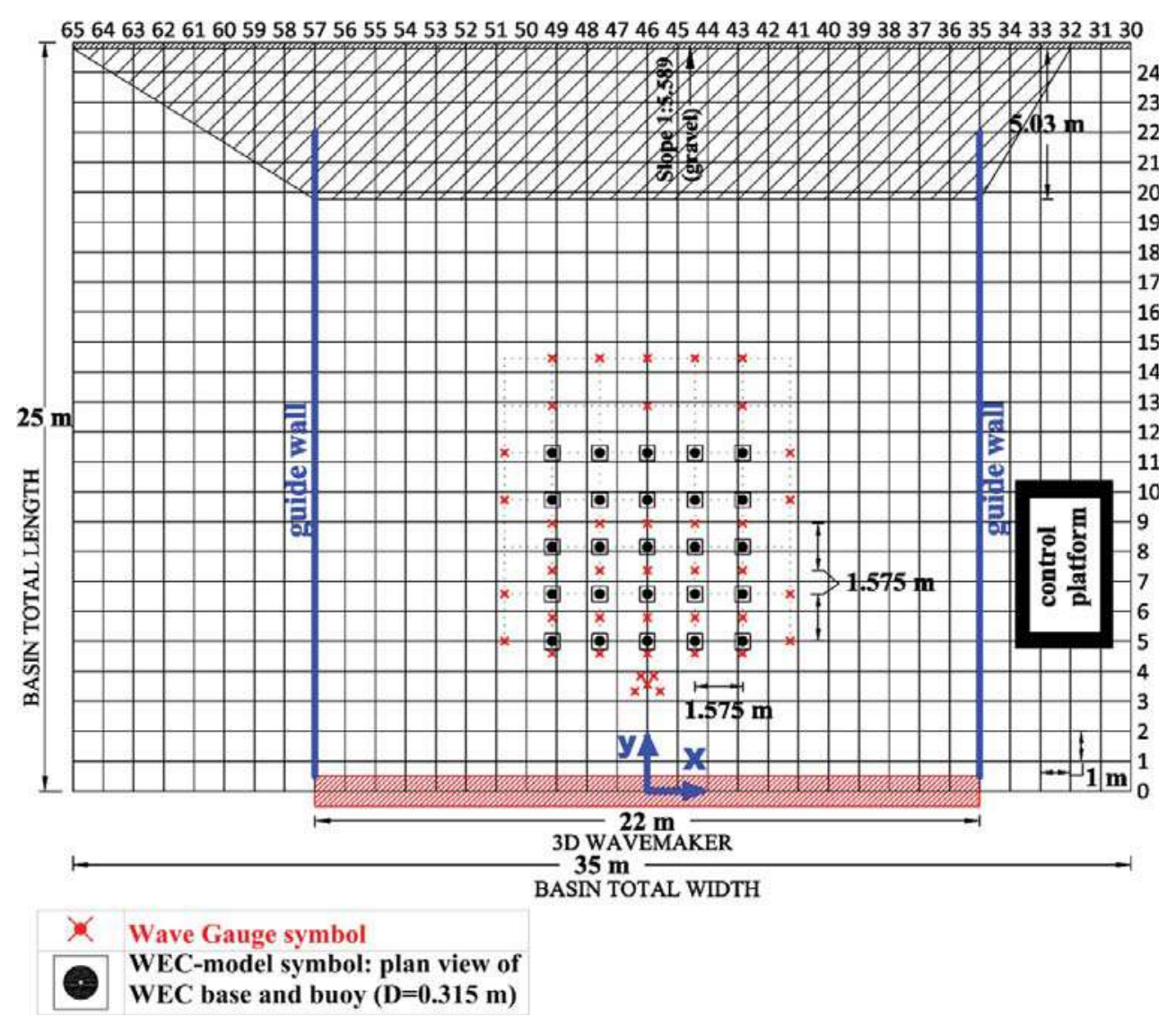

FIG. 4. Plan view of the DHI Shallow Water Wave Basin and $5 \times 5$-WEC aligned array. Grid at $1.0 \mathrm{~m}$ increments, wave gauge arrangement $(\mathrm{x})$ and WEC positions $(\bullet)$ are indicated. The hatched region along the $\mathrm{x}$-axis at the bottom of the figure denotes the extent of the wave paddles, while at the opposite end the wave absorbing beach is shown. At the sides, plywood guide walls are used. Water depth is constant, $d_{w}=0.70 \mathrm{~m}$.

"CERC 5 wave gauge array"17 (shown in Fig. 4) is used in front of the WEC arrays for estimating wave directionality and wave reflection.

Two "WG plans" have been used throughout the experiments: (a) "WG plan 1" (Fig. 4) for recording the surface elevations around the WEC units, and (b) "WG plan 2" for recording the surface elevations at all locations where WEC units have been installed and tested within all WEC array configurations. For setting up "WG plan 2," the WEC units have been removed, and the wave gauges of "WG plan 1" have been moved to the centers of the WECs.

The undisturbed wave field has been recorded in an empty wave basin (without any WECs or support structures) using both "WG plans 1 and 2" (example of such test is shown in Fig. 1).

\section{B. Measurements of the heave displacement of the WEC units}

Each WEC unit has a potentiometer (example shown in Fig. 5(a)) which measures the time varying heave displacement. In total, 25 potentiometers have been used. The heave displacement measurements provide information on the WEC response, as well as data for calculating power absorption of the WECs. ${ }^{13-15}$

\section{Measurements of the surge force on the WEC units}

The arrangement for measuring the surge force on each WEC unit requires two load cells and is presented in Fig. 5(b). For the calculation of the surge force on a WEC unit, the sum is taken of the recorded signal at the top and the bottom load cell. The surge force has been measured on 5 WECs, situated in the central column of the WEC array geometric configurations (Fig. 4). In total, ten load cells have been used. The arrangement to measure surge forces on the WECs has been developed and constructed at the workshop of Ghent University. 

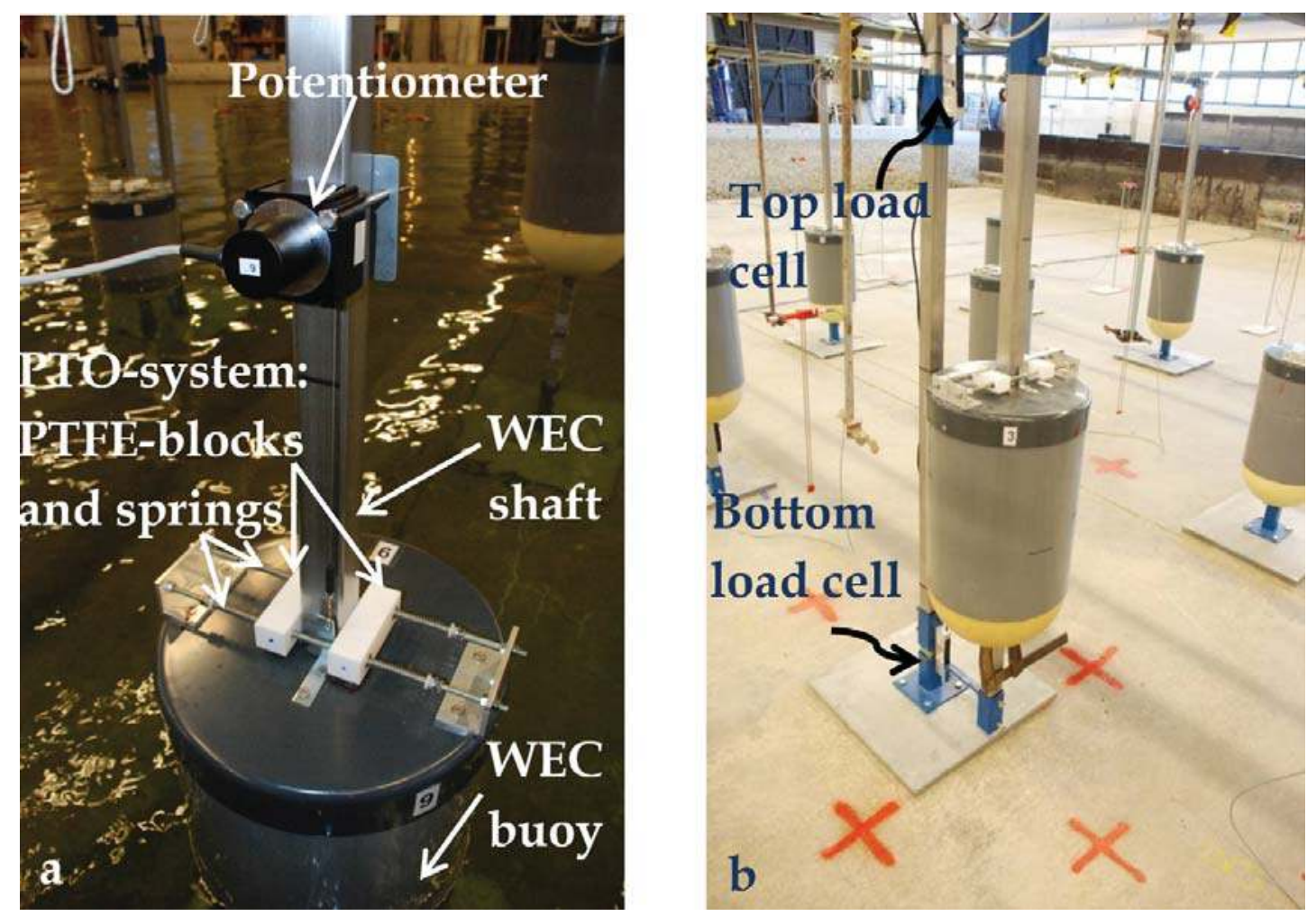

FIG. 5. (a) Potentiometer, used for heave displacement measurements of the WEC units. The PTO-system is also shown; (b) Load cells installed at the top and at the bottom of the WEC shaft, used for measurement of the surge force on the WEC units.

\section{Video acquisition}

Videos (40 fps) have been recorded for all WEC array configurations, taken from two locations outside the wave basin: (i) from a location behind the wave generator and (ii) from a location at the opposite end of wave basin, behind the wave absorbing beach.

\section{OVERVIEW OF THE “WECwakes” DATABASE AND TEST PARAMETERS}

\section{A. Database description}

During the WEC array experiments, measurements of time series of surface elevations, WEC heave displacement, and wave induced surge forces on the WEC buoys have been acquired (up to 76, simultaneously, measured data). A list of the acquired data from one experiment showing the composition of the created database is presented in Table I. Each WEC, wave gauge, force gauge, and potentiometer has been assigned a unique number. Moreover, the obtained data exhibit a variation of the test parameters listed in Table II. Finally, a wide range of different geometric lay-outs has been tested (shown in Table III). Test combinations from Tables I-III have resulted into almost 600 experiments and 45600 acquired data, which became the basis of a wide WEC array experimental database. A detailed discussion of the database characteristics is provided by Stratigaki et al. ${ }^{18}$ and background details by Stratigaki. ${ }^{15,28}$

\section{B. Methodology of the WECwakes experiments}

Several idealised wave and wave-WEC interaction problems have been considered (diffraction, radiation, and absorption) for varying WEC array configurations and wave conditions. Generic point absorber type wave energy converters have been developed for these experiments. The WECs in dry conditions are illustrated in Figure 3, where also the metal support frame is presented, used to test various combinations of WEC farm configurations in a timeeffective way. The tested WEC arrays provide experimental data suitable for studies of the interaction between both widely and closely spaced point absorber type WEC arrays. ${ }^{13,14}$

Regarding the wave conditions, the experiments are based on theoretical arguments for: (i) waves propagating through a WEC array, (ii) the dependence of wave transformation on 
TABLE I. List of acquired time series data, acquisition channels, and employed instrumentation.

\begin{tabular}{|c|c|c|c|c|}
\hline Ch. \# & Channel name & Channel content description & Units & Type of instrument employed \\
\hline 1 & Time & Time step $0.025 \mathrm{~s}$ & seconds $[\mathrm{s}]$ & - \\
\hline 2 & WG \#01 & Surface elevations at WG \#01 & meters $[\mathrm{m}]$ & DHI type resistive wave gauge \\
\hline$\cdots$ & WG \#. & Surface elevations at WG \#. & meters $[\mathrm{m}]$ & DHI type resistive wave gauge \\
\hline 42 & WG \#41 & Surface elevations at WG \#41 & meters $[\mathrm{m}]$ & DHI type resistive wave gauge \\
\hline 43 & D 1 & Wave paddle displacement & meters $[\mathrm{m}]$ & Laser measurements of paddle positions \\
\hline 44 & Thermometer & Temperature & $\begin{array}{c}\text { Degrees } \\
\text { Celsius }\left[{ }^{\circ} \mathrm{C}\right]\end{array}$ & Thermometer \\
\hline 45 & FG \#01 & Surge force at WEC \#01 (top) & Newtons $[\mathrm{N}]$ & Load Cell-Model 614-Tedea-Huntleigh \\
\hline 46 & FG \#02 & Surge force at WEC \#01 (bottom) & Newtons $[\mathrm{N}]$ & Load Cell-Model 614-Tedea-Huntleigh \\
\hline$\cdots$ & FG \#. & Surge force at WEC \#. & Newtons $[\mathrm{N}]$ & Load Cell-Model 614-Tedea-Huntleigh \\
\hline 53 & FG \#09 & Surge force at WEC \#05 (top) & Newtons $[\mathrm{N}]$ & Load Cell-Model 614-Tedea-Huntleigh \\
\hline 54 & FG \#10 & Surge force at WEC \#05 (bottom) & Newtons $[\mathrm{N}]$ & Load Cell-Model 614-Tedea-Huntleigh \\
\hline 55 & FWG & Spare channel used for testing & $\ldots$ & $\ldots$ \\
\hline 56 & Pot \#16 (DHI) & Heave displacement: WEC \#16 & meters $[\mathrm{m}]$ & DHI Ship Movement Meter \\
\hline ... & Pot \#. (DHI) & Heave displacement: WEC \#. & meters $[\mathrm{m}]$ & DHI Ship Movement Meter \\
\hline 65 & Pot \#25 (DHI) & Heave displacement: WEC \#25 & meters $[\mathrm{m}]$ & DHI Ship Movement Meter \\
\hline 66 & Pot \#01 (GENT) & Heave displacement: WEC \#01 & meters $[\mathrm{m}]$ & Draw-wire sensor model FD60; ALTHERIS \\
\hline$\ldots$ & Pot \#. (GENT) & Heave displacement: WEC \#. & meters $[\mathrm{m}]$ & Draw-wire sensor model FD60; ALTHERIS \\
\hline 79 & Pot \#14 (GENT) & Heave displacement: WEC \#14 & meters $[\mathrm{m}]$ & Draw-wire sensor model FD60; ALTHERIS \\
\hline 80 & Pot \#15 (GENT) & Heave displacement: WEC \#15 & meters $[\mathrm{m}]$ & Draw-wire sensor model AD160; ALTHERIS \\
\hline
\end{tabular}

geometrical and operational properties of the WECs which compose the array, and (iii) the dependence of wave transformation on the lateral, $w$, and longitudinal, $l$, spacing between the WECs. The methodology includes regular (with varying wave propagation angles), polychromatic, irregular, and short-crested waves propagating through WEC arrays.

The reflection, transmission, and absorption of an individual WEC unit have been first experimentally determined, as well as decay characteristics (no wave action). Additional WECs have been gradually added in the set-up, eventually resulting in large WEC arrays.

The experiments have focussed on the acquisition of the following data:

(1) Wave height, as primary output, around and within the WEC array.

(2) Measurements of undisturbed wave field, around and within the WEC array and at the locations (centres) of the wave energy converters.

(3) Measurements of diffracted wave (only) and perturbed wave field, due to WECs under incident wave conditions. Measurements have been taken around and within the WEC array.

TABLE II. Overview of the test parameters' variation throughout the "WECwakes" experiments.

\begin{tabular}{lc}
\hline \hline Parameter & Range \\
\hline wave period, $T=T p[\mathrm{~s}]$ & $0.87-1.51$ \\
wave height, $H(H m 0)[\mathrm{m}]$ & $0.018-0.104$ \\
spacing between WECs $(\mathrm{D}=0.315 \mathrm{~m})[\mathrm{m}]$ & $5 \mathrm{D}, 10 \mathrm{D}$ (pairs: up to 20D) \\
WEC number within a configuration [-] & $0-25$ \\
WEC motion & free decay, fixed, damped response, free response \\
PTO: spring compression, $d x[\mathrm{~mm}]$ & $0,50.5,45.5,40.5,35.5,30.5,20.5,10.5$ \\
wave type $[-]$ & Regular, polychromatic, irregular long- \& short-crested \\
angle of incident waves $[\mathrm{deg}]$ & $0,10,20$ \\
short-crestedness, $s_{\text {max }}[-]$ & $0,10,75$ \\
\hline \hline
\end{tabular}


TABLE III. Short overview of the tested WEC (array) configurations and the WECwakes database characteristics. Tests with an individual WEC, 2-WEC columns/rows, and 5-WEC columns/rows have been executed with WECs at various locations in the wave basin. ${ }^{\mathrm{a}}$

\begin{tabular}{|c|c|c|c|c|}
\hline \multicolumn{4}{|c|}{ Sketches of tested WEC (array) configurations } & \multirow{2}{*}{$\begin{array}{l}\text { Database characteristics at a glance } \\
\text { Simultaneous measurements of: WEC } \\
\text { response, forces on WECs and wave field. }\end{array}$} \\
\hline Configuration & $\begin{array}{l}\text { Layout } \\
\text { sketch }\end{array}$ & Configuration & $\begin{array}{l}\text { Layout } \\
\text { sketch }\end{array}$ & \\
\hline Waves (no WECs) & N/A & 10-WEC column & $\begin{array}{l}: \cdots: \\
0 \cdots:\end{array}$ & $\begin{array}{l}\text { Wide range of parameter variations: } \\
\text { o large number of combinations } \\
\text { o hundreds of tests with varying: }\end{array}$ \\
\hline Individual WEC & 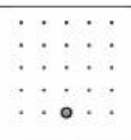 & 5x5-WEC aligned & $\begin{array}{lllll}0 & 0 & 0 & 0 & 0 \\
0 & 0 & 0 & 0 & 0 \\
0 & 0 & 0 & 0 & 0 \\
0 & 0 & 0 & 0 & 0 \\
0 & 0 & 0 & 0 & 0\end{array}$ & $\begin{array}{l}\text { - array lay-out, } \\
\text { - WEC motion, } \\
\text { - wave conditions. }\end{array}$ \\
\hline 2-WEC column & $\begin{array}{l}\therefore \\
\therefore \\
\therefore \therefore\end{array}$ & $5 \times 5-W E C$ staggered & 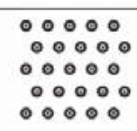 & $\begin{array}{l}\text { - wave types. } \\
\text { othousands of different time series for: }\end{array}$ \\
\hline 2-WEC row & $\begin{array}{ll}\therefore & \vdots \\
\therefore & \vdots \\
\therefore & \vdots\end{array}$ & $\begin{array}{l}3 \times 3-W E C \text { aligned } \\
10 \mathrm{D}\end{array}$ & 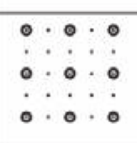 & $\begin{array}{l}\text { - WEC heave displacement } \\
\text { - surface elevations } \\
\text { - surge forces }\end{array}$ \\
\hline 5-WEC column & $\begin{array}{lll}\cdots & 0 \\
\cdots & 0 & 0 \\
\vdots & 0 & 0 \\
\cdots & 0\end{array}$ & $\begin{array}{l}3 \times 3-W E C \text { aligned } \\
5 \mathrm{D}\end{array}$ & 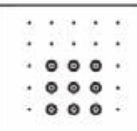 & $\begin{array}{l}\text { Database applicability: } \\
\text { - WEC arrays, }\end{array}$ \\
\hline 5-WEC row & 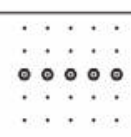 & 13-WEC staggered & $\begin{array}{llll}0 & 0 & 0 \\
0 & 0 & 0 & 0 \\
0 & 0 & 0 & 0 \\
0 & 0 & 0 & 0 \\
0 & 0 & 0 & 0\end{array}$ & $\begin{array}{l}\text { floatıng structures/platforms, } \\
\text { fixed cylinders, ... }\end{array}$ \\
\hline
\end{tabular}

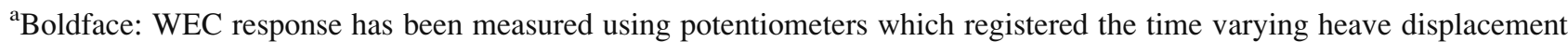
of each WEC buoy. The wave induced surge forces on WECs have been measured using load cells. The wave field measurements consist of surface elevation measurements by a network of 41 resistive wave gauges.

(4) Measurements of wave field in front of the WEC arrays providing data for the incident wave field.

(5) Measurements of the response of all WECs of the array (recording of the WEC heave displacement).

(6) Measurements of the wave induced surge forces on the WECs used for estimating WEC array power absorption and wave impact on the WECs.

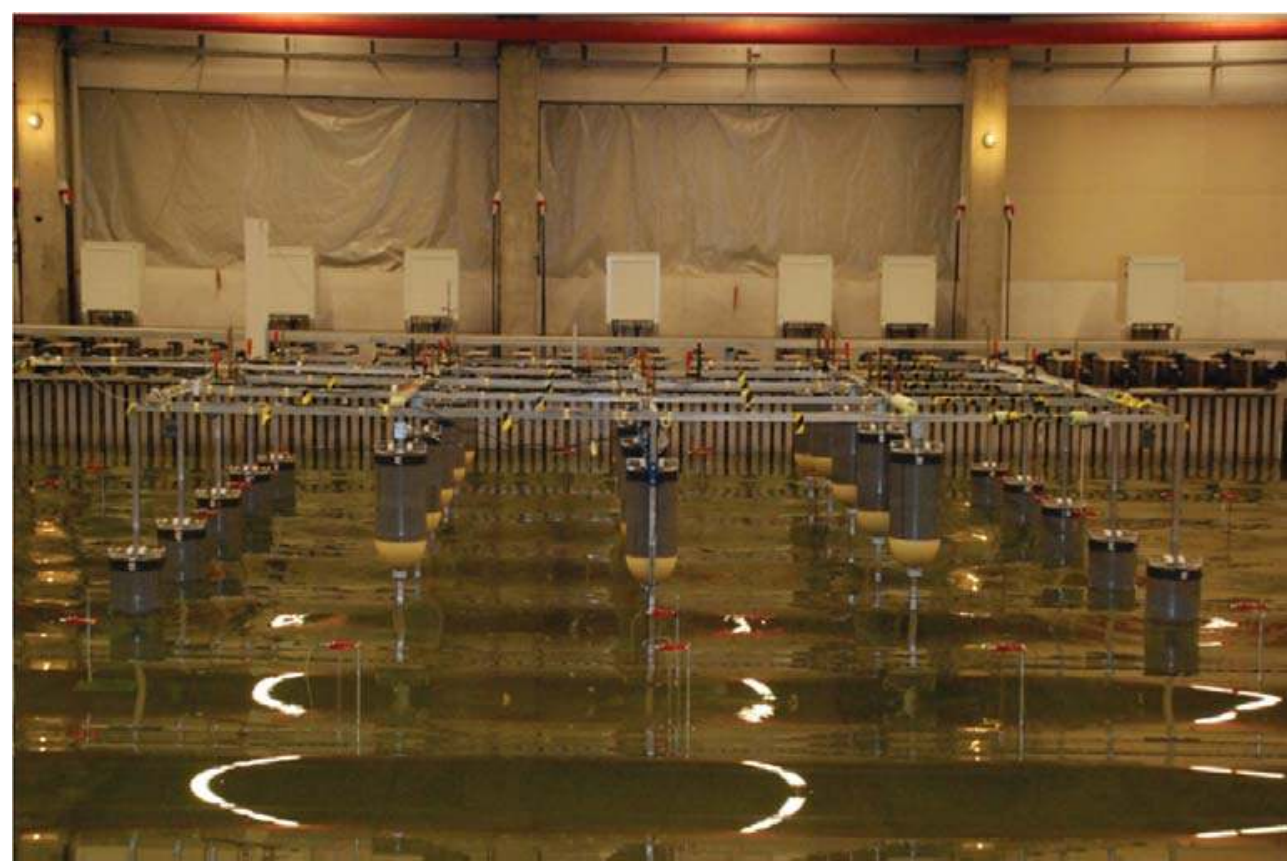

FIG. 6. A 10-WEC 2-column array (WECs \#21-\#25 and WECs \#16-\#20) under regular waves with $\theta=0^{\circ}$. The other 15 WEC units are held stationary above the water surface. View from behind the wave absorbing beach. 


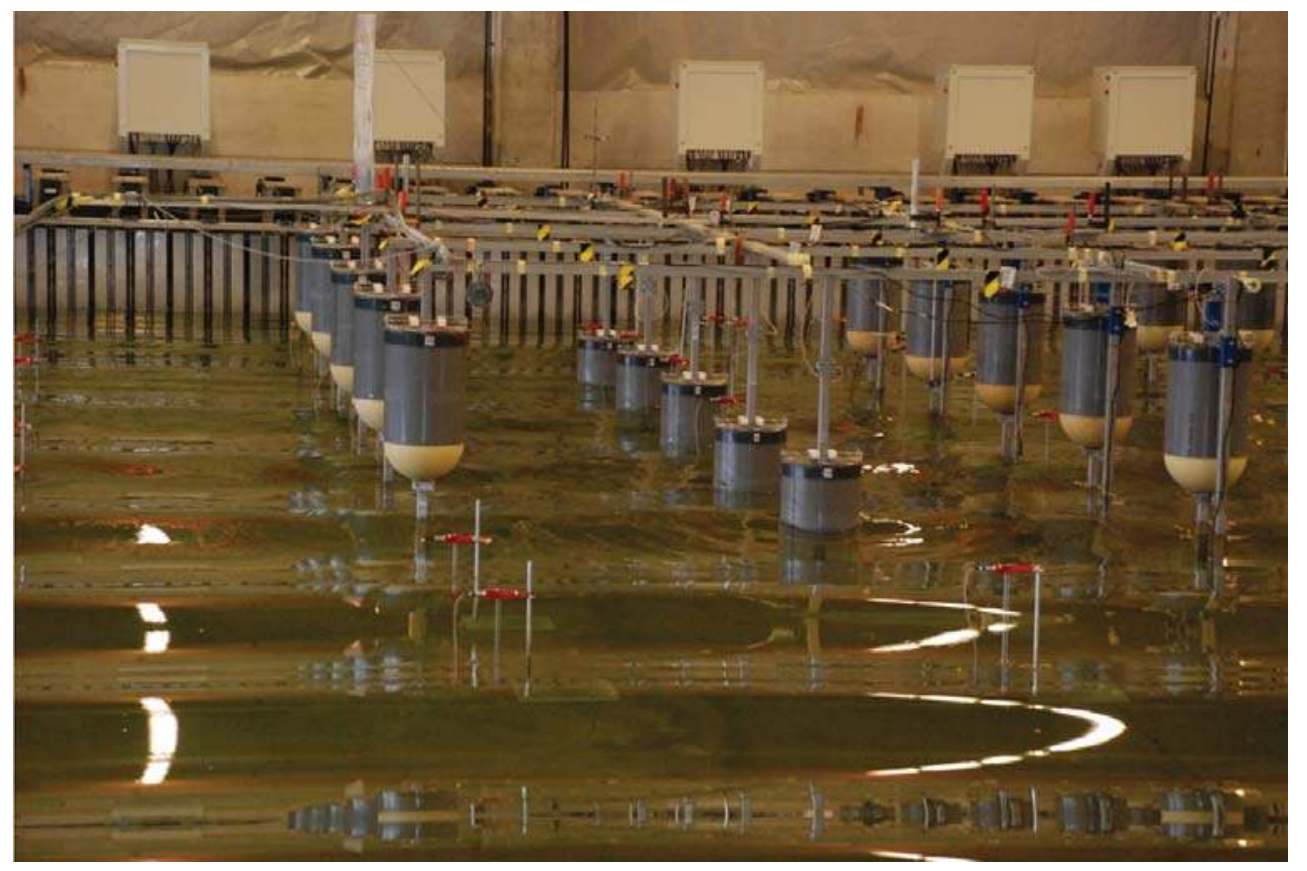

FIG. 7. A 5-WEC column array (WECs \#06-\#10) under regular waves with $\theta=0^{\circ}$. The other 20 WEC units are held stationary above the water surface. View from behind the wave absorbing beach.

Indicative illustrations of tested WEC array configurations and wave conditions during the WECwakes project are presented in Figures 6 and 7.

\section{RESULTS OF POWER OUTPUT AND WAVE FIELD MODIFICATION AROUND $5 \times 5$-WEC ARRAYS}

\section{A. Quantification of extra-array effects}

An objective of the WECwakes project was to study the effect of WEC arrays on the wave field. The wave field modifications due to wave energy extraction and the WECs' motion have been quantified around the $5 \times 5$-WEC arrays (presented in Table III), of aligned and staggered lay-out, respectively, for unidirectional irregular waves $\left(T_{p}=1.26 \mathrm{~s}\right.$ and $\left.H_{m 0}=0.104 \mathrm{~m}\right)$.

To measure the combined incident-diffracted-radiated wave field (or else the perturbed wave field), due to the heaving response of the WECs, damping has been applied through the PTO-system. All test results presented in this paper have been executed using a damping by spring compression increment $\mathrm{dx}=30.5 \mathrm{~mm}$ at the PTO-system, corresponding to optimal power absorption conditions. ${ }^{15}$ A difference percentage $(\times 100 \%)$ term is defined in Eq. (1) and plotted in Figs. 8 (a) and 8 (b) for the $5 \times 5$-WEC arrays, used for quantifying the effect of the
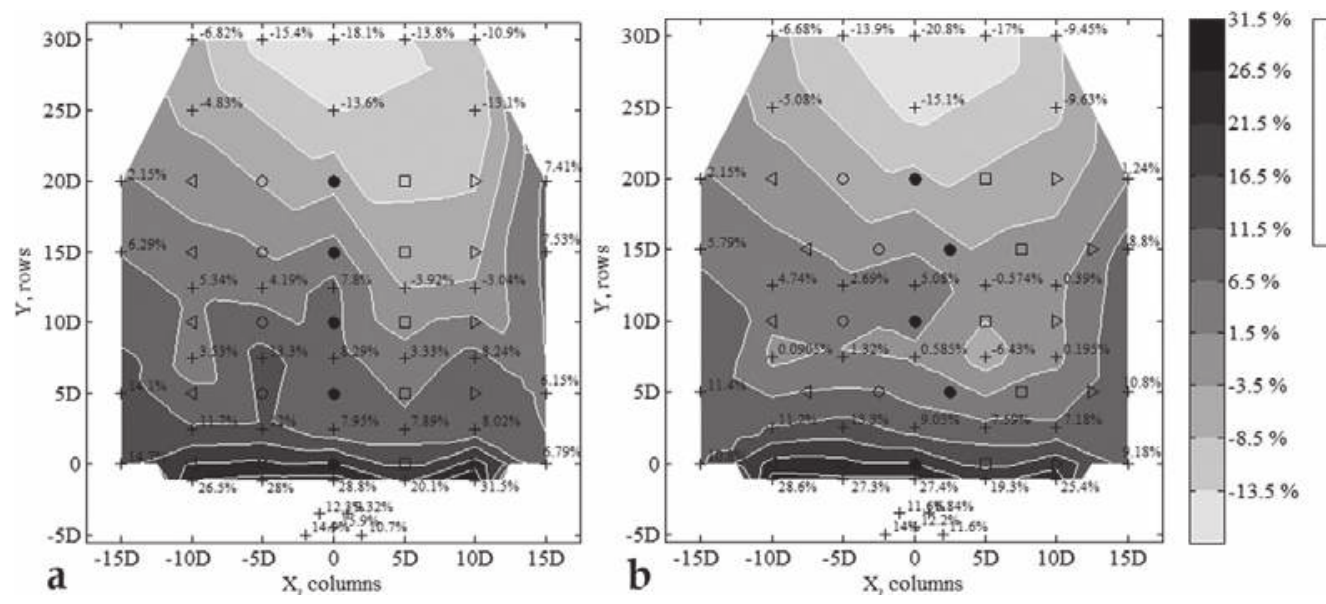

FIG. 8. Non-dimensional percentage of change of $H_{m 0}$ at locations within and around the $5 \times 5$-WEC aligned (a) and the staggered (b) array due to perturbed wave field (heaving WECs with damping applied). Unidirectional irregular waves of $T_{p}=1.26 \mathrm{~s}$ and $H_{m 0}=0.104 \mathrm{~m}$. The basin width (X, columns) and length (Y, rows) are expressed in number of WEC unit diameters, $D=0.315 \mathrm{~m}$. 
oscillating WECs under wave action (causing the perturbed wave field), on the recorded undisturbed wave field

$$
\frac{\text { perturbed wave field }- \text { recorded undisturbed wave field }}{\text { recorded undisturbed wave field }} \times 100 \% \text {. }
$$

Note that the difference percentages are positive, when the effect of the heaving WECs causes increase of the perturbed wave field amplitudes compared with the undisturbed incident wave field. On the other hand, negative difference percentages indicate wave amplitude attenuation due to the heaving WECs, compared with the undisturbed incident wave field. The negative differences, therefore, presented in Fig. 8, refer to a decrease of the significant wave height, $H_{m 0}$, due to wave power extraction by the WEC units.

In Fig. 8, a plan view of the $5 \times 5$-WEC aligned and staggered arrays is presented, where the location of the 41 wave gauges using the " + " symbol is shown. Moreover, the location of the WECs is shown, indicated by a different symbol per WEC column (see figure legend). The contour plots show the non-dimensional percentage of change of $H_{m 0}$ across the wave basin within and around the arrays due to perturbed wave field. The non-dimensional percentage of change of $H_{m 0}$ is presented with a step of $5.0 \%$. The dark grey areas correspond to increase of the significant wave height, $H_{m 0}$, while the light grey areas show decrease of $H_{m 0}$ and therefore wave height attenuation.

As presented in Figures 8(a) and 8(b) for the perturbed wave field, there is clearly wave height attenuation in the lee of the WEC arrays due to the operation of the heaving WECs, which absorb energy from the incoming waves. A large wave height decrease of up to $20.80 \%$ is found downwave of the staggered array and up to $18.10 \%$ for the aligned array. A very localised wave height increase upwave of the front row of five WECs is observed, which reaches $28.60 \%$ and $31.50 \%$ for the staggered and the aligned array, respectively. This larger wave height dissipation compared with that caused by the aligned array, is also explained by the results for power output of the $5 \times 5$-WEC staggered array. The staggered array results in higher absolute time-averaged power output and higher WEC array interaction factor compared with the aligned array under the same wave conditions.

Regarding the effect on the recorded undisturbed wave field caused by the perturbed wave field around the WEC arrays, the zone where wave height increase is observed for irregular longcrested waves is less wide for the staggered array compared with the aligned array. Wave height decrease starts between the second and the third row of 5 WECs for long-crested waves, while for the aligned array it is observed after the third row of WECs. The maximum percentages found for wave height decrease and increase when looking upwave or downwave of the staggered array are observed in front of the first row of WECs or within the CERC 5 WG array and at locations with coordinates $((0,30 D)$; $(-10 D,-1.5 D))$, respectively. For the aligned array, the maximum percentages found for wave height decrease and increase upwave or downwave of the array are observed at locations with coordinates $((0,30 \mathrm{D})$; $(10 \mathrm{D},-1.5 \mathrm{D}))$, respectively.

Moreover, wave height increase higher than $12.10 \%$ is observed within a zone limited to the WGs upwave of the front row of 5 WECs (the first WECs facing the incoming waves), due to diffraction effects.

Note that the differences presented in Figures 8(a) and 8(b) for wave height increase between $8.00 \%$ and $31.50 \%$ are progressing, almost "parallel" to the front WEC row towards the opposite end of the wave basin (landwards). When wave height attenuation starts to take over, this pattern becomes diagonal towards the WEC columns located at the sides of the WEC array. This wave pattern, which is similar for both arrays, is a result of the increased wave height at the sides of the arrays, reaching up to the fifth WEC row. Most importantly, the highest wave height dissipation is observed at locations, at least at distance $10 \mathrm{D}$ downwave of both WEC arrays, and within a zone of width $-5 D<\mathrm{X}<+5 D$.

\section{B. Quantification of intra-array interactions}

The WEC array interaction factor- $\bar{q}$-factor-as described in literature ${ }^{2,19-22}$ is a measure that quantifies the effect of intra-array interactions on the power absorption of a WEC array. 
The interaction factor is the ratio of the total power from the entire WEC array to that of the same number of WECs in isolation. If all the WECs are geometrically and operationally identical, as in the present research, the WEC array interaction $\bar{q}$-factor [-] is

$$
\bar{q}-\text { factor }=\frac{\sum_{i=1}^{N} P_{i, t o t}}{N x P_{0, t o t}},
$$

where $P_{0, t o t}$ represents the total power absorbed by an individual WEC unit; $P_{i, t o t}$ represents the power absorbed by the $i$ WEC in an array, and $N$ the number of WECs in the array. When $\bar{q}$ factor is higher than unity ( $\bar{q}$-factor $>1.0)$, the total WEC array power averaged per WEC unit in the array, is greater than the power of an individual WEC unit (in isolation). In this case, intra-array interactions have a constructive effect on the power absorption of the entire WEC array. When $\bar{q}$-factor is smaller than unity $(\bar{q}$-factor $<1.0)$, the total WEC array power averaged per WEC unit in the array is less than the power of an individual WEC unit (in isolation). In this case, intra-array interactions have a destructive effect on the power absorption of the entire WEC array.

The total power absorption, $P_{t o t}(t)$, has been estimated for the presented WEC arrays of Fig. 8. Surge force, $F_{\text {surge }}(t)$, measurements have been acquired along the central WEC column. For calculating $P_{\text {tot }}(t)$, the wave induced surge force, $F_{\text {surge }}(t)$, on WECs of the same row is considered to be equal to the measured surge force, $F_{\text {surge }}(t)$, on the respective WEC unit from the central column. By applying Eq. (2), the $\bar{q}$-factor is estimated to be 1.03 and 1.16 for the aligned and the staggered $5 \times 5$-WEC array, respectively. More detailed results on, e.g., the row-by-row and column-by-column variation of interaction factors are presented by Stratigaki. ${ }^{15}$

\section{DISCUSSION}

Large farms of 25 individual WECs are shown to have a significant effect on the resulting wave field downwave, which, for practical wave energy applications, can influence neighbouring activities in the sea, coastal eco-systems, the coastline, and the coastal defence parameters. There is clearly wave attenuation in the lee of the WEC arrays due to the operation of the oscillating WEC units. Results for extra-array effects for long-crested irregular waves (Figure 8 ) indicate up to $18.1 \%$ and $20.8 \%$ reduction in significant wave height downwave of the $5 \times 5$-WEC aligned and staggered array, respectively, for the perturbed wave field under irregular unidirectional waves of $T_{p}=1.26 \mathrm{~s}$. Thus, the staggered configuration results in around $15.0 \%$ higher wave height attenuation downwave of the staggered WEC array for long-crested irregular waves, compared with the aligned array. A large, very localised wave height increase, reaching $28.6 \%$ and $31.5 \%$ is found upwave of the front row of five WECs for the staggered and the aligned array, respectively. This larger wave height dissipation, compared with that caused by the aligned array, is also explained by the results for power output of the $5 \times 5$ WEC staggered array for irregular long-crested waves. Higher time-averaged power absorption and higher WEC array interaction factor, by almost $13.00 \%$ is achieved by the staggered array compared with the aligned array, under the same wave conditions.

Regarding the dimensions of WEC array effects:

- Wave height decrease is observed only after the third row of 5 WECs for the aligned array. For the staggered array, wave height decrease starts between the second and the third row of 5 WECs for long-crested waves.

- The highest wave height increase is found upwave of the front row of 5 WECs for both arrays (the first ones facing the incoming waves), as a result of significant diffraction effects.

- Wave height increase has a "parallel" pattern to the front WEC row. This pattern becomes diagonal towards the WEC columns located at the sides of both WEC arrays, and is a result of increased wave height at the sides of the array. 
- The highest wave height dissipation is observed at locations, at least at distance $10 D$ downwave of the WEC array, and within a zone of width $-5 D<\mathrm{X}<+5 D$.

This wave height attenuation is expected to be larger in operational conditions, since the WEC units used for the presented WEC array experiments were not optimally controlled in irregular seas; in real wave farm applications WECs are designed to be optimally controlled to extract a great amount of power from the waves, and therefore the array will cause larger wave height dissipation downwave. Wave height attenuation found that downwave of arrays can be further used for estimating the coastline evolution due to the presence of the devices, i.e., by using morphological models or by applying traditional formulae predicting the long-shore sediment transport and erosion or accretion, based on wave height parameters, e.g., as performed by Mendoza et al. ${ }^{23}$ and Nørgaard and Andersen. ${ }^{24}$

Moreover, power results show that intra-array interactions depend on the geometrical arrangement of the array, similarly to the extra-array effects. Results from interaction factors for long-crested irregular waves indicate that the intra-array interactions have a slightly constructive (by $3.0 \%$ ) effect on the overall power absorption of the entire $5 \times 5$-WEC aligned array, presented here. These intra-array interactions become, though, significantly constructive (by 16.0\%) when the second and fourth row of WECs has been shifted to form the staggered WEC array lay-out. This result is also explained by the higher power extraction by the incident waves, depicted on Fig. 8(b) by the higher wave attenuation in the lee of the staggered WEC array. Therefore, the staggered array is identified as a more effective WEC array configuration.

A detailed analysis of the environmental impact and the power output of the tested WEC arrays during the "WECwakes" project is presented by Stratigaki, ${ }^{15}$ as well as recommendations and a first series of guidelines for design of WEC arrays have been derived, based also on the existing literature. In this comprehensive analysis, wave attenuation and power results show that extra-array effects and intra-array interactions can be either positive or negative depending on the geometrical arrangement of the array, the spacing between the WECs, the number of the devices within an array, and the wave conditions. Regarding the power results, agreement is found with numerical and experimental studies performed by others, ${ }^{9,25,26}$ similarly to the wave field findings.

Most importantly, the data obtained from these experiments will be very useful to validate and extend a large range of numerical models employed to simulate response, power absorption, and wave field modifications due to oscillating WECs (or other floating structures). Such data, dealing with large wave farms, are not available in the literature. Validation of such models will enable optimization of the geometrical layout of WEC arrays for real applications and will therefore enable reduction of the cost of energy from wave energy systems (similarly to the case study demonstrated by Beels et $a{ }^{27}{ }^{27}$ ). Moreover, the WECwakes database is extensive, and can be related by others to similar applications and extrapolated to floating structures/platforms, fixed cylinders under wave action, etc., for understanding of, e.g., wave impact on the cylinders and wave field modifications around them.

Finally, this research proves the necessity for WEC concept developers to take into account the WEC array effects which are present even for large spacings between the devices (e.g., $10 \mathrm{D}$, similar to Babarit ${ }^{2}$ ) and not only focus on the optimization of individual devices.

\section{ACKNOWLEDGMENTS}

This work has been supported by the European Community's Seventh Framework Programme through the grant to the budget of the Integrating Activity HYDRALAB IV within the Transnational Access Activities, Contract No. 261520. The project is a consortium of the 7 European partners represented by the institutions affiliated 1 to 7 and coordinated by Ghent University-Belgium (Peter Troch; Vasiliki Stratigaki). The project team would like to thank the staff of DHI (including Jens Kirkegaard) for their support and the researchers from all partners institutions involved with the conduct of the experiments.

In addition, the authors would like to acknowledge the Queen's Marine Laboratory-QML (N. Ireland, UK), Flanders Hydraulics Research (Antwerp, Belgium), and from Ghent University 
(Ghent, Belgium) the Department of Civil Engineering (wave flume) and the Department of Materials Science and Engineering (tensile testing machine) for providing their experimental facilities for tests with WEC models, during the preparatory phase of the WECwakes research project. We would also like to acknowledge The Structural Design of Wave Energy Devices project (SDWED). The construction of the WEC models at the workshop of Ghent University has been funded by the Research Foundation Flanders (FWO)-Contract No. FWO-KAN-15 $23712 \mathrm{~N}$ and the further data analysis by the FWO research Project 3G029114. Finally, the first author who followed up the design and execution of the experiments as part of her Ph.D., would like to acknowledge her Ph.D. funding grant by the Research Foundation Flanders, Belgium (FWO).

${ }^{1}$ P. Troch, C. Beels, J. De Rouck, and G. De Backer, "Wake effects behind a farm of wave energy converters for irregular long-crested and short-crested waves," in Proceedings of the International Conference on Coastal Engineering, Shanghai, China (2010), Vol. 32.

${ }^{2}$ A. Babarit, "On the park effect in arrays of oscillating wave energy converters," Renewable Energy 58, 68-78 (2013).

${ }^{3}$ M. Folley, A. Babarit, L. O’Boyle, B. Child, D. Forehand, K. Silverthorne, J. Spinneken, V. Stratigaki, and P. Troch, “A review of numerical modeling of wave energy converter arrays," in Proceedings of the 31 st International Conference on Offshore Mechanics \& Arctic Engineering, Rio de Janeiro, Brazil (2012).

${ }^{4}$ Y. Li and Y.-H. Yu, "A synthesis of numerical methods for modeling wave energy converter-point absorbers," Renewable Sustainable Energy Rev. 16(6), 4352-4364 (2012).

${ }^{5} \mathrm{M}$. Vantorre, R. Banasiak, and R. Verhoeven, "Modelling of hydraulic performance and wave energy extraction by a point absorber in heave," Appl. Ocean Res. 26, 61-72 (2004).

${ }^{6} \mathrm{M}$. Vantorre, "Third-order potential theory for determining the hydrodynamic forces on axisymmetric floating and submerged bodies in a forced periodic heave motion," Ph.D. thesis (Ghent University, Ghent, 1985).

${ }^{7}$ L. Marquis, M. Kramer, and P. Frigaard, "First power production figures from the wave star Roshage wave energy converter," in Proceedings of the 3rd International Conference on Ocean Energy (ICOE-2010), Bilbao (2010).

${ }^{8}$ S. Thomas, S. D. Weller, and T. J. Stallard, "Float response within an array: Numerical and experimental comparison," in Proceedings of the 2nd International Conference on Ocean Energy (ICOE), Brest, France (2008).

${ }^{9}$ S. D. Weller, T. J. Stallard, and P. K. Stansby, "Interaction factors for a rectangular array of heaving floats in irregular waves," IET Renewable Power Gener. 4(6), 628-637 (2010).

${ }^{10}$ A. Alexandre, T. J. Stallard, and P. K. Stansby, "Transformation of wave spectra across a line of wave devices," in Proceedings of the 8th European Wave and Tidal Energy Conference, Uppsala, Sweden (2009).

${ }^{11}$ I. Ashton, L. Johanning, and B. Linfoot, "Measurement of the effect of power absorption in the lee of a wave energy converter," in Proceedings of the 28th International Conference on Offshore Mechanics \& Arctic Engineering, Honolulu, Hawaii, OMAE 2009 (2009), Vol. 4, p. 1021.

${ }^{12}$ M. Folley and T. Whittaker, "Preliminary cross-validation of wave energy converter array interactions," in Proceedings of the OMAE2013 Conference, Nantes, France (2013).

${ }^{13}$ V. Stratigaki, P. Troch, T. Stallard, J. P. Kofoed, M. Benoit, G. Mattarollo, A. Babarit, D. Forehand, and M. Folley, "Large scale experiments on farms of heaving buoys for investigation of wake dimensions, near-field and far-field effects," in Proceedings of the International Conference on Coastal Engineering 2012 (ICCE2012), Santander, Spain (2012).

${ }^{14}$ V. Stratigaki, P. Troch, M. Vantorre, M. Folley, T. Stallard, D. Forehand, J. P. Kofoed, A. Babarit, and M. Benoit, "Development of a point absorber wave energy converter for investigation of wake effects and wave energy absorption," in Proceedings of the 4th International Coastlab12 Conference, Ghent, Belgium (2013).

${ }^{15}$ V. Stratigaki, "Experimental study and numerical modelling of intra-array interactions and extra-array effects of wave energy converter arrays," Ph.D. thesis (Ghent University, Ghent, Belgium, 2014).

${ }^{16}$ P. Troch, V. Stratigaki, T. Stallard, J. P. Kofoed, D. Forehand, M. Folley, M. Benoit, A. Babarit et al. "Physical modelling of an array of 25 heaving wave energy converters to quantify variation of response and wave conditions," in Proceedings the 10th European Wave and Tidal Energy Conference Series (EWTEC), Aalborg, Denmark, 2-5 September 2013.

${ }^{17}$ L. E. Borgman and N. N. Panicker, "Design study for a suggested wave gauge array off point mugu, California," Technical Report No. 1-14, Hydraulic Engineering Laboratory, University of California at Berkeley, Berkeley, CA, USA, 1970.

${ }^{18}$ V. Stratigaki, P. Troch, T. Stallard, D. Forehand, J. P. Kofoed, M. Folley, M. Benoit, A. Babarit, and J. Kirkegaard, "Wave basin experiments with large wave energy converter arrays to study interactions between the converters and effects on other users in the sea and the coastal area," Energies 7, 701-734 (2014).

${ }^{19} \mathrm{~K}$. Budal, "Theory of absorption of wave power by a system of interacting bodies," J. Ship Res. 21, 248-253 (1977).

${ }^{20} \mathrm{D}$. V. Evans, "Some theoretical aspects of three dimensional wave energy absorbers," in Proceedings of the 1st Symposium on Wave Energy Utilization, Gothenburg, Sweden (1979).

${ }^{21}$ J. Falnes, "Radiation impedance matrix and optimum power absorption for interacting oscillators in surface waves," Appl. Ocean Res. 2, 75-80 (1980).

${ }^{22} \mathrm{~B}$. Child and V. Venugopal, "Interaction of waves with an array of floating wave energy devices," in Proceedings of the 7th European Wave and Tidal Energy Conference, Porto, Portugal (2007).

${ }^{23}$ E. Mendoza, R. Silva, B. Zanuttigh, E. Angelelli, T. Lykke Andersen, L. Martinelli, J. Q. H. Nørgaard, and P. Ruol, "Beach response to wave energy converter farms acting as coastal defense," Coastal Eng. 87, 97 (2014).

${ }^{24}$ J. H. Nørgaard and T. Lykke Andersen, "Experimental and numerical investigation of wave transmission from a floating wave dragon wave energy converter," in Proceedings of the International Ocean and Polar Engineering Conference, Rhodes (ISOPE), Rhodes, Greece, 17-22 June 2012. 
${ }^{25}$ T. J. Stallard, P. K. Stansby, and A. Williamson, “An experimental study of closely spaced point absorber arrays," in Proceedings of the 18th International Offshore \& Polar Engineering Conference, Canada (2008).

${ }^{26}$ A. Babarit, "Impact of long separating distances on the energy production of two interacting wave energy converters," Ocean Eng. 37, 718-729 (2010).

${ }^{27}$ C. Beels, P. Troch, J. P. Kofoed, P. Frigaard, J. Vindahl Kringelum, P. Carsten Kromann, M. Heyman Donovan, J. De Rouck, and G. De Backer, "A methodology for production and cost assessment of a farm of wave energy converters," Renewable Energy 36(12), 3402-3416 (2011).

${ }^{28}$ P. Troch, V. Stratigaki, T. Stallard, D. Forehand, M. Folley, J. P. Kofoed, M. Benoit, A. Babarit, M. Vantorre, and J. Kirkegaard, "An overview of the WECwakes project: Physical modelling of an array of 25 wave energy converters," in Proceedings of the 3rd IAHR Europe Congress, Porto, Portugal, 14-16 April 2014. 